\title{
New exact solution of generalized biological population model
}

\author{
Omer Acan ${ }^{a, *}$, Maysaa Mohamed Al Qurashi ${ }^{b}$, Dumitru Baleanu ${ }^{c, d}$ \\ a Siirt University, Art and Science Faculty, Department of Mathematics, Siirt, Turkey. \\ ${ }^{b}$ King Saud University, Faculty of Art and Science, Department of Mathematics, P. O. Box 22452, Riyadh 11495, Saudi Arabia. \\ ${ }^{c}$ Cankaya University, Faculty of Art and Science, Department of Mathematics, Ankara, Turkey. \\ ${ }^{d}$ Institute of Space Sciences, Magurele-Bucharest, Romania.
}

Communicated by X.-J. Yang

\begin{abstract}
In this study, a mathematical model of the generalized biological population model (GBPM) gets a new exact solution with a conformable derivative operator (CDO). The new exact solution of this model will be obtained by a new approximate analytic technique named three dimensional conformable reduced differential transform method (TCRDTM). By using this technique, it is possible to find new exact solution as well as closed analytical approximate solution of a partial differential equations (PDEs). Three numerical applications of GBPM are given to check the accuracy, effectiveness, and convergence of the TCRDTM. In these applications, obtained new exact solutions in conformable sense are compared with the exact solutions in Caputo sense in literature. The comparisons are illustrated in 3D graphics. The results show that when $\alpha \rightarrow 1$, the exact solutions in conformable and Caputo sense converge to each other. In other cases, exact solutions different from each other are obtained. (C)2017 All rights reserved.
\end{abstract}

Keywords: Numerical solution, biological populations model, reduced differential transform method, conformable derivative, partial differential equations.

2010 MSC: 35R11, 74H15.

\section{Introduction}

Linear and non-linear fractional and non-fractional problems of differential equations play a major role in various fields such as biology, physics, chemistry, mathematics, astronomy, fluids mechanics, mathematics, and engineering. It is not always possible to find analytical solutions to these problems $[1,2,4-19,24,25,28,34,35,37,38,40,42,48-54]$. Therefore, it is very important to handle these problems appropriately and solve them or develop solutions. Recently, a new derivative called CDO was introduced and also by the help of this newly defined derivative, the behaviors of many problems have been studied and some solutions techniques have been developed $[1,3,6,7,12,14,20,21,23,29-31,47]$. In 2016, Acan et al. [6] introduced two dimensional conformable reduced differential transform method (CRDTM) based on RDTM and CDO for the PDEs. It is shown that CRDTM is an easy applicable analytical method

\footnotetext{
${ }^{*}$ Corresponding author

Email addresses: omeracan@yahoo.com (Omer Acan), maysaa@ksu.edu.sam (Maysaa Mohamed Al Qurashi), dumitru@cankaya.edu.tr (Dumitru Baleanu)
}

doi:10.22436/jnsa.010.07.44 
and gives the exact solution for PDEs. We will extend this method, which is called TCRDTM, to three dimensions. This method is used to find new exact solution of the GBPM in conformable sense as described below.

Scientists in biology believe that migration or scattering is very important in regulating species populations. In a region $\Omega$, the diffusion of species is defined by the three position parameters $\vec{\xi}=(x, y)$ and time $t$ that are population density (PD) $\rho(\vec{\xi}, t)$, the population supply $\psi(\vec{\xi}, t)$, and diffusion velocity (DV) $v(\vec{\xi}, t)$ [27]. The $\rho(\vec{\xi}, t)$ gives the individual number per unit volume at time $t$ and position $\vec{\xi}$; over any sub-region $G$ of $\Omega$, the integral $\rho(\vec{\xi}, t)$ gives all population of the region $G$ at time $t . \psi(\vec{\xi}, t)$ presents the rate at which individuals are supplied, per unit volume, at position $\vec{\xi}$ by births and deaths. The DV $v(\vec{\xi}, t)$ shows the average velocity belonging to the individuals who occupy the position $\vec{\xi}$ at time $t$, and it reports the population flow from point to point. The entities $\rho(\vec{\xi}, t), v(\vec{\xi}, t)$, and $\psi(\vec{\xi}, t)$ must obey the following population balance law, for any regular sub-region $G$ of $\Omega$ and for any time $t$

$$
\frac{d^{\alpha}}{d t^{\alpha}} \int_{G} \rho d V+\int_{\partial G} \rho \vec{v} \cdot \hat{m} d A=\int_{G} \psi d V,
$$

where $\hat{m}$ is the outward unit normal to the boundary $\partial G$ of $G$. In (1.1), the derivative has been taken in the conformable derivative sense. By the assumptions [33]

$$
\psi=\psi(\rho) \text { and } v=-\mu(\rho) \nabla \rho,
$$

where $\nabla$ is the Laplace operator and $\mu(\rho)>0$ for $\rho>0$, two-dimensional non-linear degenerate parabolic PDEs for the PD $\rho$ can be obtained as

$$
\frac{\partial^{\alpha}}{\partial t^{\alpha}} \rho(x, y, t)=\frac{\partial^{2}}{\partial x^{2}} \omega(\rho)+\frac{\partial^{2}}{\partial y^{2}} \omega(\rho)+\psi(\rho), x, y \in R, t \in[0, \infty), 0<\alpha \leqslant 1 .
$$

The statement (1.2) is BPM. Gurney and Nisbet [26] employed a method, for a special case for the modeling of the animals' population. Model (1.2) with $\omega(\rho)=\rho^{2}$, is the following equation

$$
\frac{\partial^{\alpha}}{\partial t^{\alpha}} \rho(x, y, t)=\frac{\partial^{2}}{\partial x^{2}} \rho^{2}+\frac{\partial^{2}}{\partial y^{2}} \rho^{2}+\psi(\rho), x, y \in R, t \in[0, \infty), 0<\alpha \leqslant 1,
$$

subject to the initial condition (IC) $\rho(x, y, 0)$. For $\alpha=1,(1.3)$ reduces to the standard biological population model

$$
\frac{\partial}{\partial t} \rho(x, y, t)=\frac{\partial^{2}}{\partial x^{2}} \rho^{2}+\frac{\partial^{2}}{\partial y^{2}} \rho^{2}+\psi(\rho), x, y \in R, t \in[0, \infty) .
$$

Some properties of (1.3) such as holder estimates and its solutions have been studied in [33]. The constitutive equations for $\psi(\rho)$ can be given as

(i) $\psi(\rho)=k \rho$, k constant, Malthusian law [27];

(ii) $\psi(\rho)=k_{1} \rho-k_{2} \rho^{2}, k_{1}, k_{2}$ positive constants, Verhulst law [33];

(iii) $\psi(\rho)=k \rho^{\gamma},(k>0,0<\gamma \leqslant 1)$, Porous media [36].

Let us deal with a more general form of $\psi(\rho)$ as $\psi(\rho)=h \rho^{\eta}\left(1-\mu \rho^{\tau}\right)$ so that (1.3) becomes

$$
\frac{\partial^{\alpha}}{\partial t^{\alpha}} \rho(x, y, t)=\frac{\partial^{2}}{\partial x^{2}} \rho^{2}+\frac{\partial^{2}}{\partial y^{2}} \rho^{2}+h \rho^{\eta}\left(1-\mu \rho^{\tau}\right), x, y \in R, t \in[0, \infty), 0<\alpha \leqslant 1,
$$

where $h, \eta, \tau, \mu \in R$. If $h=k, \eta=1, \mu=0$ and $h=k_{1}, \eta=\tau=1, \mu=\frac{k_{2}}{k_{1}}$, then (1.4) leads to Malthusian law and Verhulst law, respectively. The above mentioned models have been a research topic for many researchers and scientists. Different methods and derivative definitions have been used to explore these models $[13,22,26,27,32,33,36,39,41,43-46]$. 
In this study, we propose a new exact solution for GBPM. TCRDTM, based on CDO and RDTM, is used to obtain this new solution. The obtained new exact solutions in conformable sense are compared with the exact solutions [46] in Caputo sense for some $\alpha$ values. The comparisons of these exact solutions are illustrated in 3D graphics. For this, in Section 2, we give basic definitions and important properties of CDO. In Section 3, two dimensions CRDTM has been extended to three dimensions CRDTM (TCRDTM). For this new method some definitions and theorems are given. In Section 4, some applications for GBPM are given. And in the final section, we give the conclusion.

\section{On the conformable derivative operator}

Definition $2.1([1,12,30])$. Given a function $f:[0, \infty) \rightarrow \mathbb{R}$, then the conformable derivative (CD) of $f$ of order $\alpha$ is defined by:

$$
\left(T_{\alpha} f\right)(t)=\frac{d^{\alpha}}{d t^{\alpha}} f(t)=\lim _{\varepsilon \rightarrow 0} \frac{f\left(t+\varepsilon t^{1-\alpha}\right)-f(t)}{\varepsilon}
$$

for all $t>0, \alpha \in(0,1]$.

Lemma $2.2([1,12,30])$. Let $\mathrm{f}, \mathrm{g}$ be $\alpha$-differentiable at a point $\mathrm{t}>0$ for $\alpha$. Then

(i) $\mathrm{T}_{\alpha}(\mathrm{af}+\mathrm{bg})=\mathrm{a}\left(\mathrm{T}_{\alpha} \mathrm{f}\right)+\mathrm{b}\left(\mathrm{T}_{\alpha} \mathrm{g}\right)$ for all $\mathrm{a}, \mathrm{b} \in \mathbb{R}$ and $\alpha \in(0,1]$;

(ii) $\mathrm{T}_{\alpha}(\mathrm{f}(\mathrm{t}))=0$ for constant function $\mathrm{f}(\mathrm{t})=\lambda, \alpha \in(0,1]$;

(iii) $T_{\alpha}(f g)=f\left(T_{\alpha} g\right)+g\left(T_{\alpha} f\right), \alpha \in(0,1]$;

(iv) $T_{\alpha}(f / g)=\frac{g\left(T_{\alpha} f\right)-f\left(T_{\alpha} g\right)}{g^{2}}, \alpha \in(0,1]$;

(v) if $\mathrm{f}$ is $\mathrm{n}$ times differentiable at $\mathrm{t}$, then $\mathrm{T}_{\alpha}(\mathrm{f}(\mathrm{t}))=\mathrm{t}^{\lceil\alpha\rceil-\alpha} \mathrm{f}^{(\lceil\alpha\rceil)}(\mathrm{t}), \alpha \in(\mathrm{n}, \mathrm{n}+1]$, where $\lceil\alpha\rceil$ is the smallest integer greater than or equal to $\alpha$.

Lemma 2.3 ([1]). Suppose that $\mathrm{f}$ is infinitely $\alpha$-differentiable function for $\alpha \in(0,1]$ at a neighborhood of a point $\mathrm{t}_{0}$. Then $\mathrm{f}$ has the conformable power series expansion

$$
f(t)=\sum_{k=0}^{\infty} \frac{\left(T_{\alpha}^{(k)} f\right)\left(t_{0}\right)\left(t-t_{0}\right)^{\alpha k}}{\alpha^{k} k !}, t_{0}<t<t_{0}+R^{1 / \alpha}, R>0 .
$$

Here $\left(T_{\alpha}^{(k)} f\right)\left(t_{0}\right)$ denotes the application of the $C D$ for $k$ times.

\section{Three dimensional conformable reduced differential transform method}

In 2016, Acan et al. [6] introduced the two dimensional CRDTM. In this section, we will extend this method to three dimensions. Throughout this study, the lowercase $u(x, y, t)$ represents the original function while the uppercase $U_{k}^{\alpha}(x, y)$ stands for three dimensional conformable reduced differential transformed (TCRDT) function. The basic definitions of TCRDTM are presented as follows.

Definition 3.1. Assume that $u(x, y, t)$ is analytic and differentiated continuously with respect to three variables $x, y, t$ in its domain. The TCFRDT of $u(x, y, t)$ is defined as

$$
u_{k}^{\alpha}(x, y)=\frac{1}{\alpha^{k} k !}\left[\left(T_{\alpha}^{(k)} u\right)\right]_{t=t_{0}}
$$

where $0<\alpha \leqslant 1, \alpha$ is a parameter describing the order of conformable derivative,

$$
T_{\alpha}^{(k)} \mathfrak{u}=\underbrace{\left(T_{\alpha} T_{\alpha} \cdots T_{\alpha}\right)}_{k \text { times }} u(x, y, t)
$$

and the $t$ dimensional spectrum function $\mathrm{u}_{\mathrm{k}}^{\alpha}(x, y)$ is the TCRDT function. 
Definition 3.2. Let $u_{k}^{\alpha}(x, y)$ be the TCRDT of $u(x, y, t)$. Inverse TCRDT of $u_{k}^{\alpha}(x, y)$ is defined as

$$
u\left(x_{1}, x_{2}, \ldots, x_{n}, t\right)=\sum_{k=0}^{\infty} u_{k}^{\alpha}(x, y)\left(t-t_{0}\right)^{\alpha k}=\sum_{k=0}^{\infty} \frac{1}{\alpha^{k} k !}\left[T_{\alpha}^{(k)} u\right]_{t=t_{0}}\left(t-t_{0}\right)^{\alpha k}
$$

TCRDT of initial conditions for integer order derivatives are defined as

$$
u_{k}^{\alpha}(x, y)=\left\{\begin{array}{ll}
\frac{1}{(\alpha k) !}\left[\frac{\partial^{\alpha k}}{\partial t^{\alpha k}} u(x, y, t)\right]_{t=t_{0}}, & \text { if } \alpha k \in \mathbb{Z}^{+}, \\
0, & \text { if } \alpha k \notin \mathbb{Z}^{+},
\end{array} \text {for } k=0,1,2, \ldots,\left(\frac{m}{\alpha}-1\right),\right.
$$

where $m$ is the order of conformable PDE.

Theorem 3.3. Let $\mathrm{a}$ and $\mathrm{b}$ be constants. If $\mathrm{u}(x, y, t)=\mathrm{a} v(x, y, t) \pm b w(x, y, t)$, then $\mathrm{u}_{\mathrm{k}}^{\alpha}(x, y)=a V_{k}^{\alpha}(x, y) \pm$ $\mathrm{bW}_{\mathrm{k}}^{\alpha}(x, y)$.

Proof. CFRDT of $v(x, y, t)$ and $w(x, y, t)$ can be written as

$$
V_{k}^{\alpha}(x, y)=\frac{1}{\alpha^{k} k !}\left[T_{\alpha}^{(k)} v\right]_{t=t_{0}}, \quad W_{k}^{\alpha}(x, y)=\frac{1}{\alpha^{k} k !}\left[T_{\alpha}^{(k)} w\right]_{t=t_{0}}
$$

Because of Lemma 2.2 (i),

$$
\mathrm{u}_{\mathrm{k}}^{\alpha}(x, y)=\frac{1}{\alpha^{\mathrm{k}} \mathrm{k} !}\left[\mathrm{T}_{\alpha}^{(\mathrm{k})}(\mathrm{a} v \pm \mathrm{b} w)\right]_{\mathrm{t}=\mathrm{t}_{0}}=\frac{\mathrm{a}}{\alpha^{\mathrm{k}} \mathrm{k} !}\left[\mathrm{T}_{\alpha}^{(\mathrm{k})} v\right]_{\mathrm{t}=\mathrm{t}_{0}} \pm \frac{\mathrm{b}}{\alpha^{\mathrm{k}} \mathrm{k} !}\left[\mathrm{T}_{\alpha}^{(\mathrm{k})} \mathrm{w}\right]_{\mathrm{t}=\mathrm{t}_{0}}=a V_{k}^{\alpha}(x, y) \pm \mathrm{bW} W_{k}^{\alpha}(x, y)
$$

The proof is completed.

Theorem 3.4. If $u(x, y, t)=v(x, y, t) w(x, y, t)$, then $u_{k}^{\alpha}(x, y)=\sum_{s=0}^{k} v_{s}^{\alpha}(x, y) W_{k-s}^{\alpha}(x, y)$.

Proof. By the help of Definition 3.2, $v(x, y, t)$ and $w(x, y, t)$ can be written as

$$
v(x, y, t)=\sum_{k=0}^{\infty} V_{k}^{\alpha}(x, y)\left(t-t_{0}\right)^{\alpha k}, \quad w(x, y, t)=\sum_{k=0}^{\infty} W_{k}^{\alpha}(x, y)\left(t-t_{0}\right)^{\alpha k} .
$$

Then, $u(x, t)$ is obtained as

$$
\begin{aligned}
u_{k}^{\alpha}(x, y)= & \sum_{k=0}^{\infty} v_{k}^{\alpha}(x, y)\left(t-t_{0}\right)^{\alpha k} \sum_{k=0}^{\infty} W_{k}^{\alpha}(x, y)\left(t-t_{0}\right)^{\alpha k} \\
= & \left(v_{0}^{\alpha}(x, y)+V_{1}^{\alpha}(x, y)\left(t-t_{0}\right)^{\alpha}+V_{2}^{\alpha}(x, y)\left(t-t_{0}\right)^{2 \alpha}+\cdots+V_{n}^{\alpha}(x, y)\left(t-t_{0}\right)^{n \alpha}+\cdots\right) \\
& \times\left(W_{0}^{\alpha}(x, y)+W_{1}^{\alpha}(x, y)\left(t-t_{0}\right)^{\alpha}+W_{2}^{\alpha}(x, y)\left(t-t_{0}\right)^{2 \alpha}+\cdots+W_{n}^{\alpha}(x, y)\left(t-t_{0}\right)^{n \alpha}+\cdots\right) \\
= & V_{0}^{\alpha}(x, y) W_{0}^{\alpha}(x, y)+\left(V_{0}^{\alpha}(x, y) W_{1}^{\alpha}(x, y)+V_{1}^{\alpha}(x, y) W_{0}^{\alpha}(x, y)\right)\left(t-t_{0}\right)^{\alpha} \\
& +\left(V_{0}^{\alpha}(x, y) W_{2}^{\alpha}(x, y)+V_{1}^{\alpha}(x, y) W_{1}^{\alpha}(x, y)+V_{2}^{\alpha}(x, y) W_{0}^{\alpha}(x, y)\right)\left(t-t_{0}\right)^{2 \alpha}+\cdots \\
= & \sum_{k=0}^{\infty} \sum_{s=0}^{k} V_{s}^{\alpha}(x, y) W_{k-s}^{\alpha}(x, y)\left(t-t_{0}\right)^{k \alpha} .
\end{aligned}
$$

Hence, $u_{k}^{\alpha}(x, y)$ is found as 


$$
u_{k}^{\alpha}(x, y)=\sum_{s=0}^{k} v_{s}^{\alpha}(x, y) W_{k-s}^{\alpha}(x, y)
$$

The proof is completed.

Theorem 3.5. If $u(x, y, t)=T_{\alpha} v(x, y, t)$, then $u_{k}^{\alpha}(x, y)=\alpha(k+1) v_{k+1}^{\alpha}(x, y)$.

Proof. TCRDT of $v(x, y, t)$ can be written as

$$
V_{k}^{\alpha}(x, y)=\frac{1}{\alpha^{k} k !}\left[T_{\alpha}^{(k)} v\right]_{t=t_{0}}
$$

for $u(x, y, t)=T_{\alpha} v(x, y, t)$,

$$
\begin{aligned}
\mathrm{u}_{\mathrm{k}}^{\alpha}(\mathrm{x}, \mathrm{y})=\frac{1}{\alpha^{\mathrm{k}} \mathrm{k} !}\left[\mathrm{T}_{\alpha}^{(\mathrm{k})}\left(\mathrm{T}_{\alpha} v\right)\right]_{\mathrm{t}=\mathrm{t}_{0}} & =\frac{1}{\alpha^{\mathrm{k}} \mathrm{k} !}\left[\mathrm{T}_{\alpha}^{(\mathrm{k}+1)} v\right]_{\mathrm{t}=\mathrm{t}_{0}} \\
& =\alpha(\mathrm{k}+1) \frac{1}{\alpha^{\mathrm{k}+1}(\mathrm{k}+1) !}\left[\mathrm{T}_{\alpha}^{(\mathrm{k}+1)} v\right]_{\mathrm{t}=\mathrm{t}_{0}}=\alpha(\mathrm{k}+1) \mathrm{V}_{\mathrm{k}}^{\alpha}(\mathrm{x}, \mathrm{y}) .
\end{aligned}
$$

The proof is completed.

Theorem 3.6. If $\mathrm{u}(\mathrm{x}, \mathrm{y}, \mathrm{t})=x^{\mathrm{p}} \mathrm{y}^{\mathrm{q}}\left(\mathrm{t}-\mathrm{t}_{0}\right)^{\mathrm{m}}$, then $\mathrm{u}_{\mathrm{k}}^{\alpha}(\mathrm{x}, \mathrm{y})=x^{\mathrm{p}} \mathrm{y}^{\mathrm{q}} \delta\left(\mathrm{k}-\frac{\mathrm{m}}{\alpha}\right)$, where $\delta(\mathrm{k})= \begin{cases}1, & \text { if } \mathrm{k}=0, \\ 0, & \text { if } \mathrm{k} \neq 0 .\end{cases}$

Proof. TCRDT of $u(x, y, t)=x^{p} y^{q}\left(t-t_{0}\right)^{m}$ is

$$
u_{k}^{\alpha}(x, y)=\frac{1}{\alpha^{k} k !}\left[T_{\alpha}^{(k)}\left(x^{p} y^{q}\left(t-t_{0}\right)^{m}\right)\right]_{t=t_{0}} .
$$

If the conformable derivative of $u(x, y, t)=x^{p} y^{q}\left(t-t_{0}\right)^{m}$, with respect to $t$, is calculated for $k$ times, where $\alpha \in(0,1]$, then

$$
u_{k}^{\alpha}(x, y)=\frac{1}{\alpha^{k} k !}\left[x^{p} y^{q}\left(m(m-\alpha) \cdots(m-(k-1) \alpha)\left(t-t_{0}\right)^{m-k \alpha}\right)\right]_{t=t_{0}}
$$

is obtained. If $k=\frac{m}{\alpha}$, then

$$
\begin{aligned}
u_{k}^{\alpha}(x, y) & =\frac{1}{\alpha^{\frac{m}{\alpha}}\left(\frac{m}{\alpha}\right) !} x^{p} y^{q}\left(m(m-\alpha) \cdots\left(m-\left(\frac{m}{\alpha}-1\right) \alpha\right)\left(t-t_{0}\right)^{m-k \alpha}\right) \\
& =\frac{1}{\alpha^{\frac{m}{\alpha}}\left(\frac{m}{\alpha}\right) !} x^{p} y^{q}(m(m-\alpha) \cdots(\alpha))=\frac{\alpha^{\frac{m}{\alpha}}}{\alpha^{\frac{m}{\alpha}}\left(\frac{m}{\alpha}\right) !} x^{p} y^{q}\left(\frac{m}{\alpha}\left(\frac{m}{\alpha}-1\right) \cdots(1)\right)=x^{p} y^{q} .
\end{aligned}
$$

Otherwise, for $t=t_{0}$,

$$
u_{k}^{\alpha}(x, y)=0,
$$

hence

$$
u_{k}^{\alpha}(x, y)=x^{p} y^{q} \delta\left(k-\frac{m}{\alpha}\right)
$$

is obtained. The proof is completed.

\section{Numerical applications}

Three applications are considered in this section. All of the results are calculated by MAPLE program. 
Example 4.1. First, we consider the following non-linear BPM [46]:

$$
\frac{\partial^{\alpha}}{\partial t^{\alpha}} \rho(x, y, t)=\frac{\partial^{2}}{\partial x^{2}} \rho^{2}+\frac{\partial^{2}}{\partial y^{2}} \rho^{2}+h \rho, 0<\alpha \leqslant 1
$$

subject to the IC

$$
\rho(x, y, 0)=\sqrt{x y} .
$$

The exact solution in Caputo sense of the non-linear BPM (4.1) is given as [46]:

$$
\rho(x, y, t)=\sqrt{x y} E_{\alpha}\left(h t^{\alpha}\right)
$$

where $E_{\alpha}\left(h t^{\alpha}\right)$ is the Mittag-Leffler function, defined as $E_{\alpha}(\zeta)=\sum_{k=0}^{\infty} \frac{\zeta^{k}}{\Gamma(1+k \alpha)}$.

Now solve this problem by using TCRDTM. By taking the TCRDT of (4.1), it can be obtained that

$$
\alpha(k+1) P_{k+1}^{\alpha}(x, y)=\frac{\partial^{2}}{\partial x^{2}}\left[\sum_{s=0}^{k} P_{s}^{\alpha}(x, y) P_{k-s}^{\alpha}(x, y)\right]+\frac{\partial^{2}}{\partial y^{2}}\left[\sum_{s=0}^{k} P_{s}^{\alpha}(x, y) P_{k-s}^{\alpha}(x, y)\right]+h P_{k}^{\alpha}(x, y),
$$

where $P_{k}^{\alpha}(x, y)$ is the TCRDT function. From IC (4.2) we write

$$
P_{0}^{\alpha}(x, y)=\sqrt{x y} .
$$

From (4.4) and (4.5), we can obtain the following $P_{k}^{\alpha}(x, y)$ values

$$
P_{1}^{\alpha}(x, y)=\sqrt{x y} \frac{h}{\alpha}, P_{2}^{\alpha}(x, y)=\sqrt{x y} \frac{h^{2}}{2 ! \alpha^{2}}, \cdots, P_{m}^{\alpha}(x, y)=\sqrt{x y} \frac{h^{m}}{m ! \alpha^{m}}, \cdots .
$$

Then, from (4.6), the set of values $\left\{P_{k}^{\alpha}(x)\right\}_{k=0}^{m}$ gives us the following approximate result

$$
\tilde{\rho}_{m}(x, y, t)=\sum_{k=0}^{m} P_{k}^{\alpha}(x, y) t^{k \alpha}=\sum_{k=0}^{m} \sqrt{x y} \frac{h^{k}}{k ! \alpha^{k}} t^{k \alpha}
$$

From (4.7) we obtain

$$
\rho(x, y, t)=\lim _{m \rightarrow \infty} \tilde{\rho}_{m}(x, y, t)=\sqrt{x y} e^{h \frac{t^{\alpha}}{\alpha}} .
$$

The obtained analytical approximate solution given in (4.8) is a new exact solution in conformable sense for the non-linear BPM in (4.1).

Remark 4.2. If we take $\alpha=1$, then Example 4.1 is reduced to standard biological population model

$$
\frac{\partial}{\partial t} \rho(x, y, t)=\frac{\partial^{2}}{\partial x^{2}} \rho^{2}+\frac{\partial^{2}}{\partial y^{2}} \rho^{2}+h \rho
$$

with IC

$$
\rho(x, y, 0)=\sqrt{x y} .
$$

Our new exact solution (4.8) in conformable sense and the exact solution (4.3) in Caputo sense imply

$$
\rho(x, y, t)=\sqrt{x y} e^{h t} .
$$

This result is the exact solution of the standard problem in the literature. 
Now, we can compare the new exact solution in conformable sense and the exact solution [46] in Caputo sense for the non-linear BPM (4.1). For some $\alpha$ values, the comparison of exact solutions in conformable and Caputo sense are illustrated by 3D graphics in Figs. 1 and 2. The MAPLE codes for graph drawings are given in Table 1.

Table 1: The MAPLE codes for graphics.
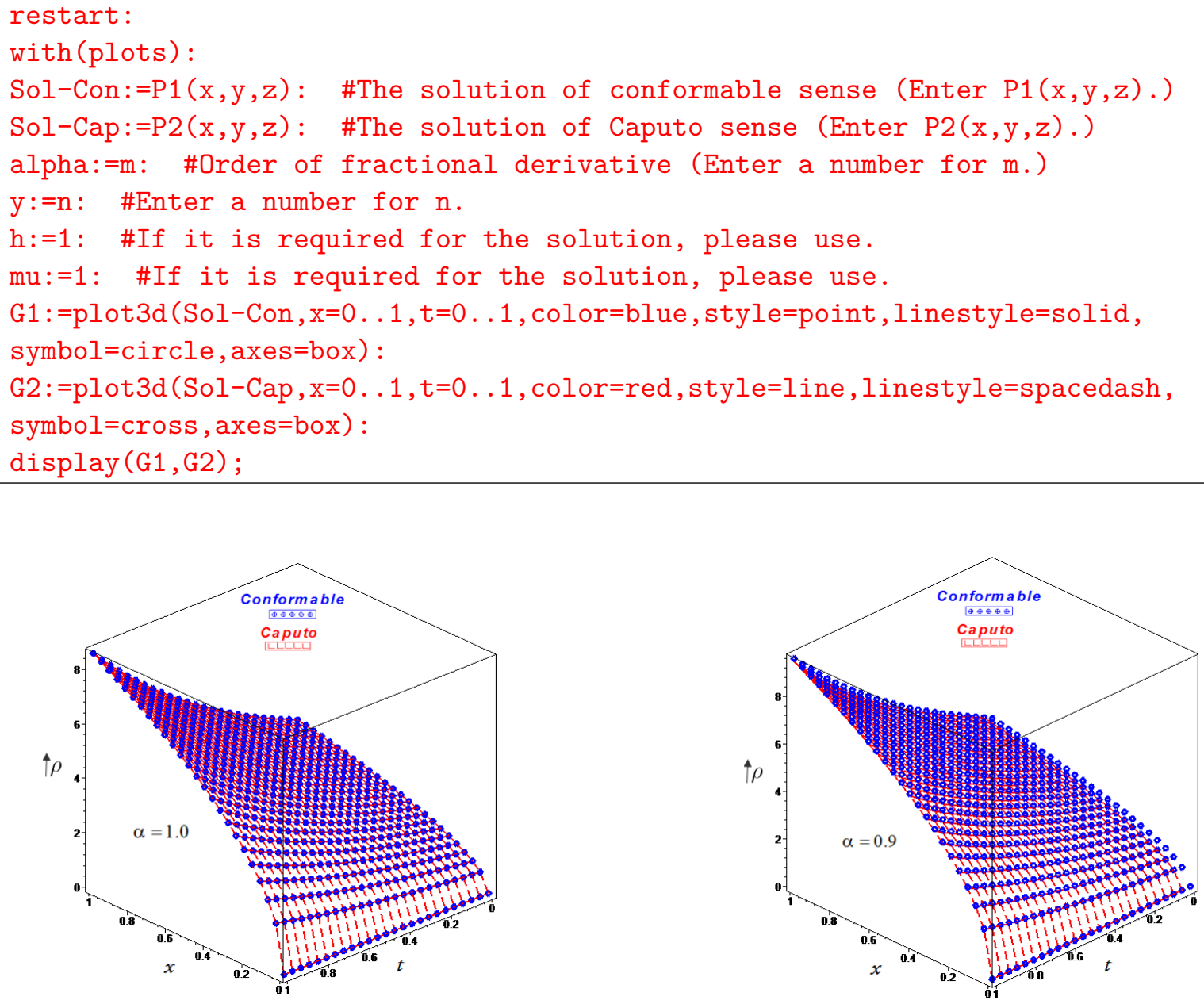

Figure 1: The plots show the comparison of exact solutions of (4.1) in conformable and Caputo sense [46] for $y=10, \mathrm{~h}=1$, and some $\alpha$ values.

Example 4.3. Second, let us consider the following non-linear BPM [46]

$$
\frac{\partial^{\alpha}}{\partial t^{\alpha}} \rho(x, y, t)=\frac{\partial^{2}}{\partial x^{2}} \rho^{2}+\frac{\partial^{2}}{\partial y^{2}} \rho^{2}+\rho, 0<\alpha \leqslant 1
$$

subject to the IC

$$
\rho(x, y, 0)=\sqrt{\sin x \sinh y} .
$$

The exact solution in Caputo sense of non-linear BPM (4.9) is given as [46]

$$
\rho(x, y, t)=\sqrt{\sin x \sinh y} E_{\alpha}\left(t^{\alpha}\right),
$$

where $E_{\alpha}\left(t^{\alpha}\right)$ is the Mittag-Leffler function, defined as $E_{\alpha}(\zeta)=\sum_{k=0}^{\infty} \frac{\zeta^{k}}{\Gamma(1+k \alpha)}$. 

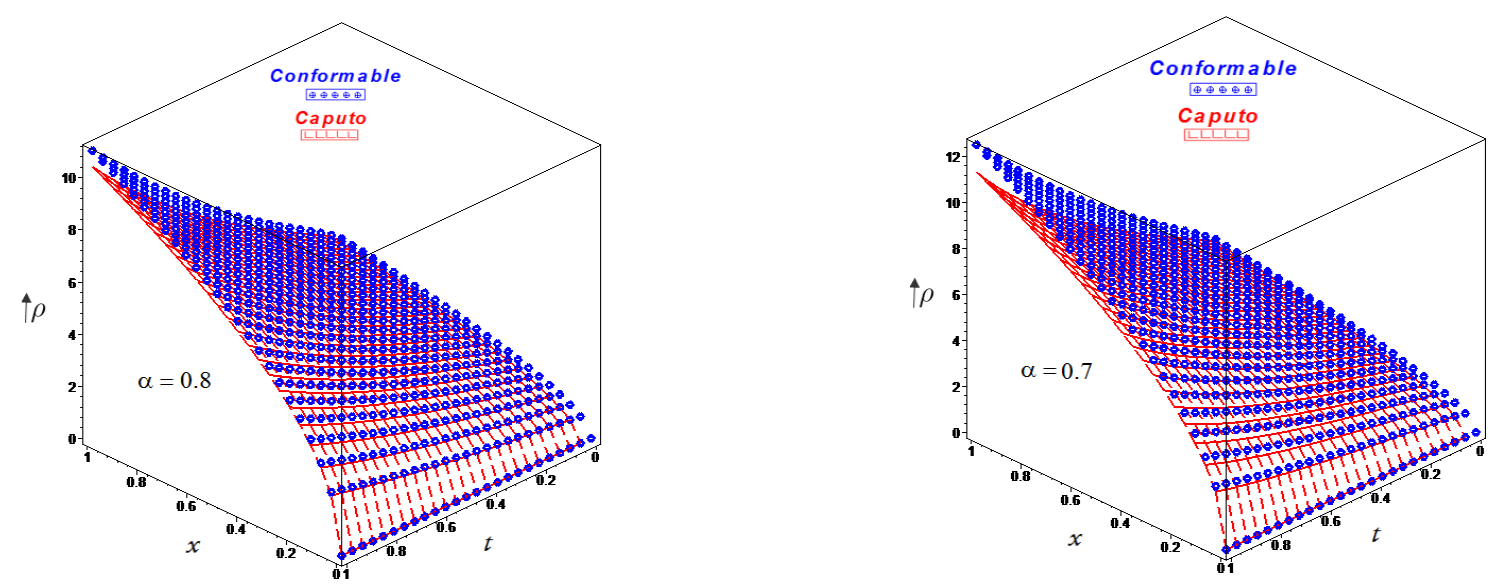

Figure 2: The plots show the comparison of exact solutions of (4.1) in conformable and Caputo sense [46] for $y=10, \mathrm{~h}=1$, and some $\alpha$ values.

Now solve this problem by using TCRDTM. By taking the TCRDT of (4.9), it can be obtained that

$$
\alpha(k+1) P_{k+1}^{\alpha}(x, y)=\frac{\partial^{2}}{\partial x^{2}}\left[\sum_{s=0}^{k} P_{s}^{\alpha}(x, y) P_{k-s}^{\alpha}(x, y)\right]+\frac{\partial^{2}}{\partial y^{2}}\left[\sum_{s=0}^{k} P_{s}^{\alpha}(x, y) P_{k-s}^{\alpha}(x, y)\right]+P_{k}^{\alpha}(x, y),
$$

where $P_{k}^{\alpha}(x, y)$ is the TCRDT function. From IC (4.10) we write

$$
P_{0}^{\alpha}(x, y)=\sqrt{\sin x \sinh y}
$$

From (4.12) and (4.13), we can obtain the following $P_{k}^{\alpha}(x, y)$ values

$$
\begin{aligned}
P_{1}^{\alpha}(x, y) & =\sqrt{\sin x \sinh y} \frac{1}{\alpha^{\prime}} \\
P_{2}^{\alpha}(x, y) & =\sqrt{\sin x \sinh y} \frac{1}{2 ! \alpha^{2}}, \\
P_{3}^{\alpha}(x, y) & =\sqrt{\sin x \sinh y} \frac{1}{3 ! \alpha^{3}}, \\
\vdots & \\
P_{m}^{\alpha}(x, y) & =\sqrt{\sin x \sinh y} \frac{1}{m ! \alpha^{m}}, \cdots
\end{aligned}
$$

Then, from (4.14), the set of values $\left\{\mathrm{P}_{\mathrm{k}}^{\alpha}(\mathrm{x})\right\}_{\mathrm{k}=0}^{\mathrm{m}}$ gives us the following approximate result

$$
\tilde{\rho}_{m}(x, y, t)=\sum_{k=0}^{m} P_{k}^{\alpha}(x, y) t^{k \alpha}=\sum_{k=0}^{m} \sqrt{\sin x \sinh y} \frac{1}{k ! \alpha^{k}} t^{k \alpha}
$$

From (4.15) we obtain

$$
\rho(x, y, t)=\lim _{m \rightarrow \infty} \tilde{\rho}_{m}(x, y, t)=\sqrt{\sin x \sinh y} e^{\frac{t^{\alpha}}{\alpha}} .
$$

The obtained analytical approximate solution given in (4.16) is a new exact solution in conformable sense for the non-linear BPM in (4.9).

Remark 4.4. If we take $\alpha=1$, then Example 4.3 is reduced to standard biological population model

$$
\frac{\partial}{\partial t} \rho(x, y, t)=\frac{\partial^{2}}{\partial x^{2}} \rho^{2}+\frac{\partial^{2}}{\partial y^{2}} \rho^{2}+\rho
$$


with IC

$$
\rho(x, y, 0)=\sqrt{\sin x \sinh y} .
$$

Our new exact solution (4.16) in conformable sense and the exact solution (4.11) in Caputo sense imply

$$
\rho(x, y, t)=\sqrt{\sin x \sinh y} e^{t}
$$

This result is the exact solution of the standard problem in the literature.

Now, we can compare the new exact solution in conformable sense and the exact solution [46] in Caputo sense for the non-linear BPM (4.9). For some $\alpha$ values, the comparison of exact solutions in conformable and Caputo sense are illustrated by 3D graphics in Figs. 3 and 4. The MAPLE codes for graph drawings are given in Table 1.
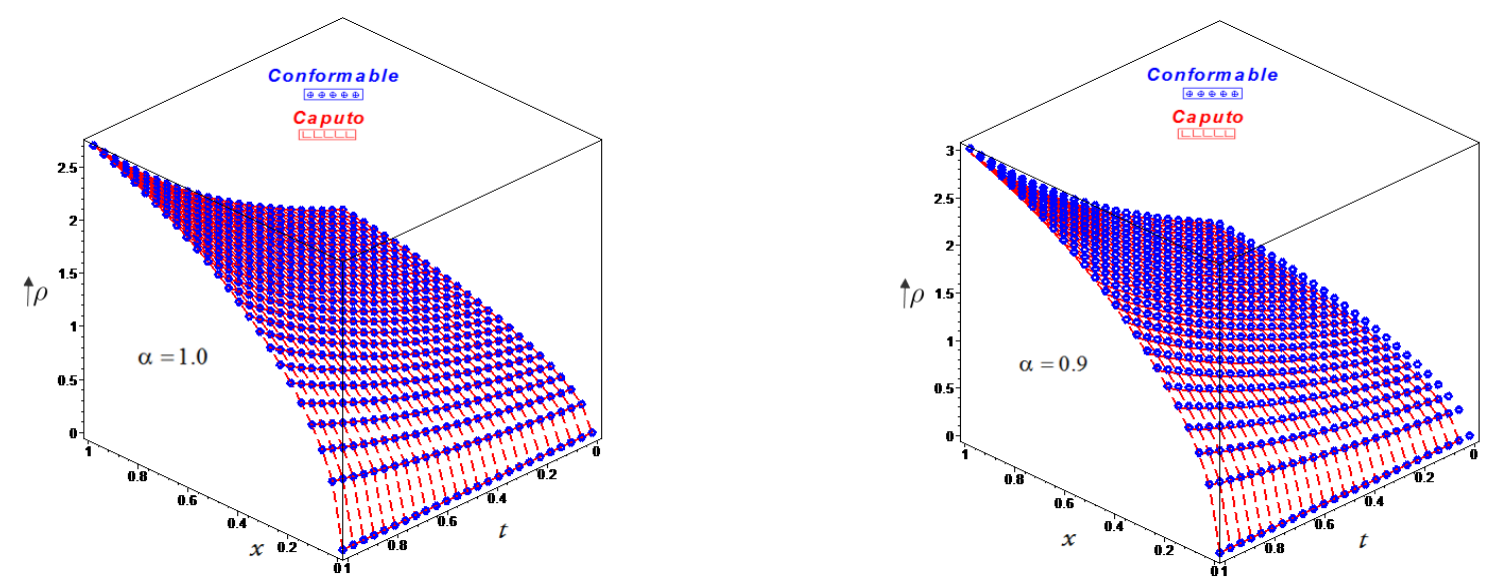

Figure 3: The plots show the comparison of exact solutions of (4.9) in conformable and Caputo sense [46] for $y=1$ and some $\alpha$ values.
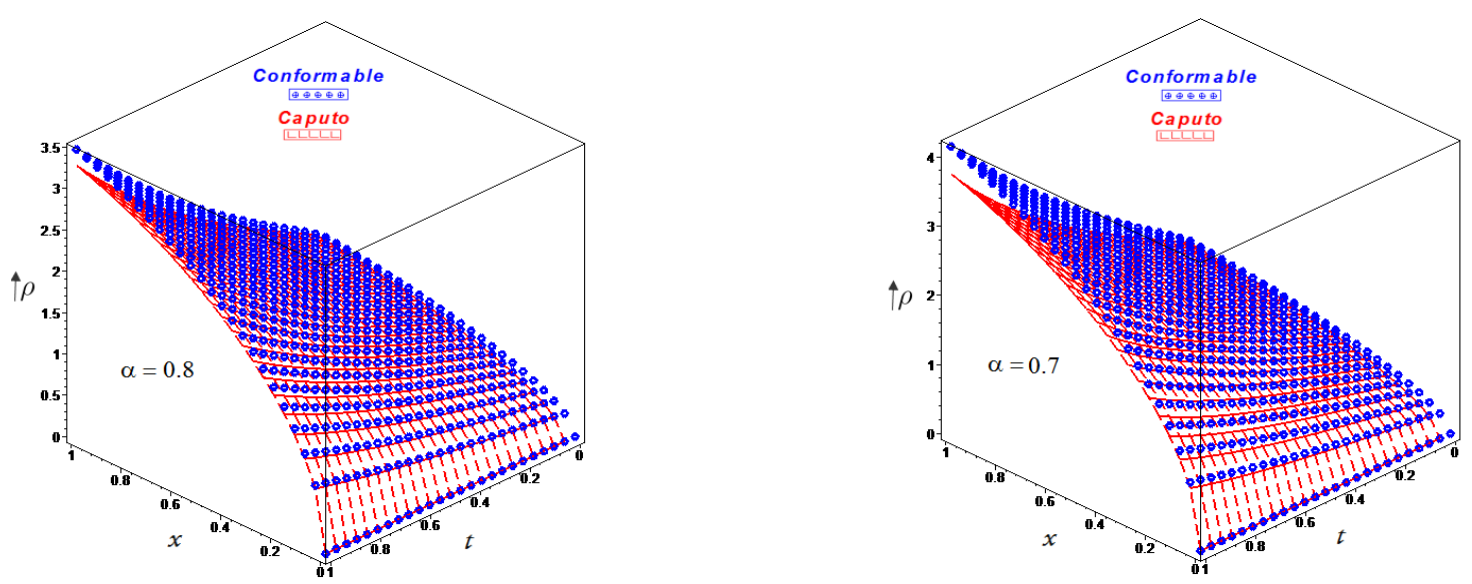

Figure 4: The plots show the comparison of exact solutions of (4.9) in conformable and Caputo sense [46] for $y=1$ and some $\alpha$ values.

Example 4.5. In the final, we discuss the following non-linear GBPM [46]:

$$
\frac{\partial^{\alpha}}{\partial t^{\alpha}} \rho(x, y, t)=\frac{\partial^{2}}{\partial x^{2}} \rho+\frac{\partial^{2}}{\partial y^{2}} \rho-\mu \rho^{2}+\rho, \quad 0<\alpha \leqslant 1
$$


subject to the IC

$$
\rho(x, y, 0)=e^{\frac{\sqrt{\mu}}{2 \sqrt{2}}(x+y)} .
$$

The exact solution in Caputo sense of non-linear GBPM (4.17) is given as [46]:

$$
\rho(x, y, t)=e^{\frac{\sqrt{\mu}}{2 \sqrt{2}}(x+y)} E_{\alpha}\left(t^{\alpha}\right),
$$

where $E_{\alpha}\left(t^{\alpha}\right)$ is the Mittag-Leffler function, defined as $E_{\alpha}(\zeta)=\sum_{k=0}^{\infty} \frac{\zeta^{k}}{\Gamma(1+k \alpha)}$.

Now solve this problem by using TCRDTM. By taking the TCRDT of (4.17), it can be obtained that

$$
\begin{aligned}
\alpha(k+1) P_{k+1}^{\alpha}(x, y)= & \frac{\partial^{2}}{\partial x^{2}}\left[\sum_{s=0}^{k} P_{s}^{\alpha}(x, y) P_{k-s}^{\alpha}(x, y)\right]+\frac{\partial^{2}}{\partial y^{2}}\left[\sum_{s=0}^{k} P_{s}^{\alpha}(x, y) P_{k-s}^{\alpha}(x, y)\right] \\
& -\mu\left[\sum_{s=0}^{k} P_{s}^{\alpha}(x, y) P_{k-s}^{\alpha}(x, y)\right]+P_{k}^{\alpha}(x, y),
\end{aligned}
$$

where $P_{k}^{\alpha}(x, y)$ is the TCRDT function. From the IC (4.18) we write

$$
P_{0}^{\alpha}(x, y)=e^{\frac{\sqrt{\mu}}{2 \sqrt{2}}(x+y)}
$$

From (4.20) and (4.21), we can obtain the following $P_{k}^{\alpha}(x, y)$ values

$$
\begin{aligned}
P_{1}^{\alpha}(x, y) & =e^{\frac{\sqrt{\mu}}{2 \sqrt{2}}(x+y)} \frac{1}{\alpha} \\
P_{2}^{\alpha}(x, y) & =e^{\frac{\sqrt{\mu}}{2 \sqrt{2}}(x+y)} \frac{1}{2 ! \alpha^{2}}, \\
P_{3}^{\alpha}(x, y) & =e^{\frac{\sqrt{\mu}}{2 \sqrt{2}}(x+y)} \frac{1}{3 ! \alpha^{3}}, \\
& \vdots \\
P_{m}^{\alpha}(x, y) & =e^{\frac{\sqrt{\mu}}{2 \sqrt{2}}(x+y)} \frac{1}{m ! \alpha^{m}}, \cdots
\end{aligned}
$$

Then, from (4.22), the set of values $\left\{P_{k}^{\alpha}(x)\right\}_{k=0}^{m}$ gives us the following approximate result

$$
\tilde{\rho}_{m}(x, y, t)=\sum_{k=0}^{m} P_{k}^{\alpha}(x, y) t^{k \alpha}=\sum_{k=0}^{m} e^{\frac{\sqrt{\mu}}{2 \sqrt{2}}(x+y)} \frac{1}{k ! \alpha^{k}} t^{k \alpha} .
$$

From (4.23) we obtain

$$
\rho(x, y, t)=\lim _{m \rightarrow \infty} \tilde{\rho}_{m}(x, y, t)=e^{\frac{\sqrt{\mu}}{2 \sqrt{2}}(x+y)+\frac{t^{\alpha}}{\alpha}} .
$$

The obtained analytical approximate solution given in (4.24) is a new exact solution in conformable sense for non-linear GBPM in (4.17).

Remark 4.6. If we take $\alpha=1$, then Example 4.5 is reduced to generalized standard biological population model

$$
\frac{\partial}{\partial t} \rho(x, y, t)=\frac{\partial^{2}}{\partial x^{2}} \rho+\frac{\partial^{2}}{\partial y^{2}} \rho-\mu \rho^{2}+\rho
$$

with IC

$$
\rho(x, y, 0)=e^{\frac{\sqrt{\mu}}{2 \sqrt{2}}(x+y)} .
$$


Our new exact solution (4.24) in conformable sense and the exact solution (4.19) in Caputo sense imply

$$
\rho(x, y, t)=e^{\frac{\sqrt{\mu}}{2 \sqrt{2}}(x+y)+t} .
$$

This result is the exact solution of the standard problem in the literature.

Now, we can compare the new exact solution in conformable sense and the exact solution [46] in Caputo sense for the non-linear GBPM (4.17). For some $\alpha$ values, the comparison of exact solutions in conformable and Caputo sense are illustrated by 3D graphics in Figs. 5 and 6. The MAPLE codes for graph drawings are given in Table 1.


Figure 5: The plots show the comparison of exact solutions of (4.17) in conformable and Caputo sense [46] for $y=1, \mu=2$, and some $\alpha$ values.
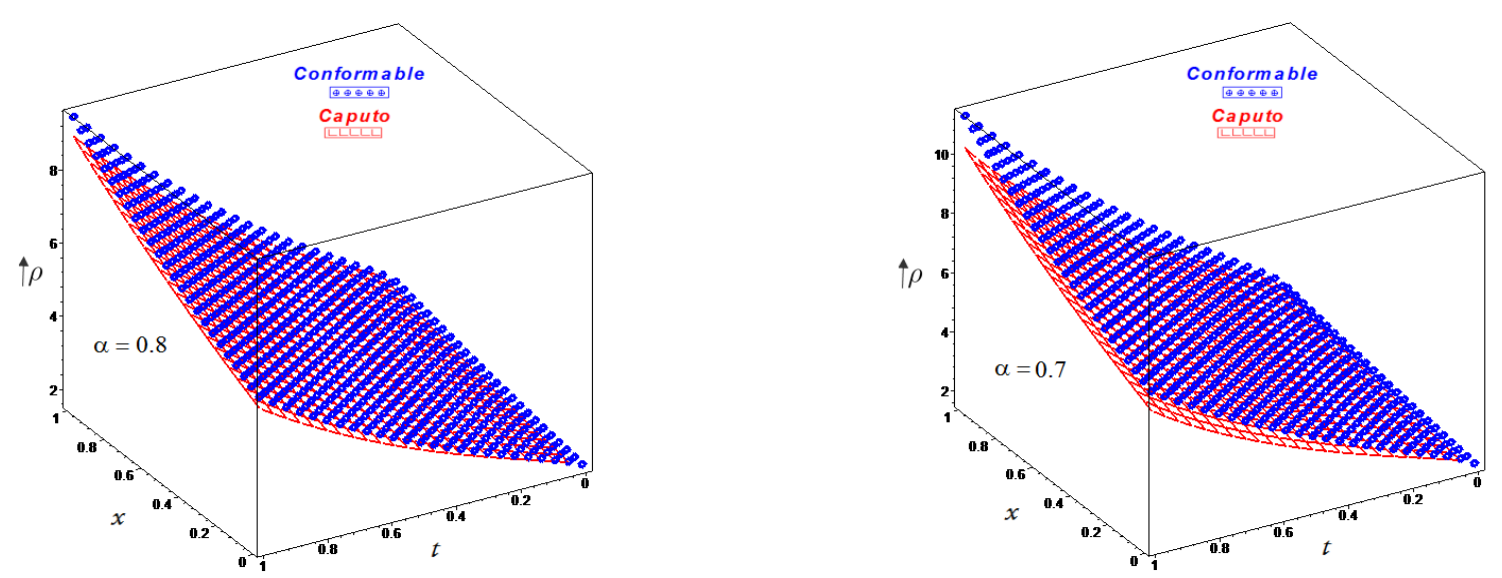

Figure 6: The plots show the comparison of exact solutions of (4.17) in conformable and Caputo sense [46] for $y=1, \mu=2$, and some $\alpha$ values.

\section{Conclusion}

In this paper, the TCRDTM is implemented in degenerate parabolic PDEs arising in the spatial diffusion biological populations. The solution obtained by the TCRDTM is an infinite power series for the appropriate IC which finds the solution without any discretization, perturbation, transformation or restrictive conditions. Three numerical examples are also illustrated considering the situations of non-linear 
phenomenon of GBPM to study the effectiveness and accurateness of the method. For each of the applications, new exact solutions with conformable sense are obtained. These new exact solutions obtained in conformable sense is compared with the exact solutions [46] in Caputo sense. For some values of $\alpha$, these comparisons are illustrated in 3D graphics. As a result of these processes, the following points on solutions can be noted as

(i) the conformable derivative produces exponential functions, while the Caputo derivative gives the Mittag Leffler functions;

(ii) when $\alpha=1$, Mittag Leffler and exponential functions in the solutions are equal to each other. When $\alpha \neq 1$, the solutions are different from each other.

Hence, when $\alpha \rightarrow 1$, the exact solutions in conformable and Caputo sense converge to the exact results of the problems in classical sense. In other cases, the solutions exhibit similar behavior, but they are not equal to each other. As it offers new conformable solutions different from Caputo solutions for these models, we hope that it gives new solutions to many problems in the branches of sciences such as physics, chemistry, biology, mathematics, and engineering.

\section{Acknowledgment}

The authors extend their appreciation to the International Scientific Partnership Program ISPP at King Saud University for funding this research work through ISPP\# 63.

\section{References}

[1] T. Abdeljawad, On conformable fractional calculus, J. Comput. Appl. Math., 279 (2015), 57-66. 1, 2.1, 2.2, 2.3

[2] O. Acan, The existence and uniqueness of periodic solutions for a kind of forced rayleigh equation, Gazi Univ. J. Sci., 29 (2016), 645-650. 1

[3] O. Acan, D. Baleanu, A new numerical technique for solving fractional partial differential equations, ArXiv, 2017 (2017), 12 pages. 1

[4] O. Acan, D. Baleanu, M. M. A. Qurashi, M. G. Sakar, Analytical approximate solutions of $(\mathrm{n}+1)$-dimensional fractal heat-like and wave-like equations, Entropy, 19 (2017), 14 pages. 1

[5] O. Acan, O. Firat, Y. Keskin, The use of conformable variational iteration method, conformable reduced differential transform method and conformable homotopy analaysis method for solving different types of nonlinear partial differential equations, Proceedings of the 3rd International Conference on Recent Advances in Pure and Applied Mathematics, Bodrum, Turkey, (2016).

[6] O. Acan, O. Firat, Y. Keskin, G. Oturanc, Solution of conformable fractional partial differential equations by reduced differential transform method, Selcuk J. Appl. Math., (2016), in press. 1, 3

[7] O. Acan, O. Firat, Y. Keskin, G. Oturanc, Conformable variational iteration method, New Trends Math. Sci., 5 (2017), 172-178. 1

[8] O. Acan, Y. Keskin, Approximate solution of Kuramoto-Sivashinsky equation using reduced differential transform method, Proc. Int. Conf. Numer. Anal. Appl. Math., AIP Publishing, 1648 (2015), 470003.

[9] O. Acan, Y. Keskin, Reduced differential transform method for $(2+1)$ dimensional type of the Zakharov-Kuznetsov ZK(n,n) equations, Proc. Int. Conf. Numer. Anal. Appl. Math., AIP Publishing, 1648 (2015), 370015.

[10] O. Acan, Y. Keskin, A comparative study of numerical methods for solving $(n+1)$ dimensional and third-order partial differential equations, J. Comput. Theor. Nanosci., 13 (2016), 8800-8807.

[11] O. Acan, Y. Keskin, A new technique of Laplace Pade reduced differential transform method for $(1+3)$ dimensional wave equations, New Trends Math. Sci., 5 (2017), 164-171.

[12] D. R. Anderson, D. J. Ulness, Newly defined conformable derivatives, Adv. Dyn. Syst. Appl., 10 (2015), 109-137. 1, $2.1,2.2$

[13] A. A. M. Arafa, S. Z. Rida, H. Mohamed, Homotopy analysis method for solving biological population model, Commun. Theor. Phys. (Beijing), 56 (2011), 797-800. 1

[14] A. Atangana, D. Baleanu, A. Alsaedi, New properties of conformable derivative, Open Math., 13 (2015), 889-898. 1

[15] D. Baleanu, A. H. Bhrawy, R. A. Van Gorder, New trends on fractional and functional differential equations, Abstr. Appl. Anal., 2015 (2015), 2 pages.

[16] D. Baleanu, K. Diethelm, E. Scalas, J. J. Trujillo, Fractional calculus, Models and numerical methods, Second edition, Series on Complexity, Nonlinearity and Chaos, World Scientific Publishing Co. Pte. Ltd., Hackensack, NJ, (2017).

[17] D. Baleanu, K. Sayevand, Performance evaluation of matched asymptotic expansions for fractional differential equations with multi-order, Bull. Math. Soc. Sci. Math. Roumanie (N.S.), 59 (2016), 3-12. 
[18] D. Baleanu, J. A. Tenreiro Machado, C. Cattani, M. C. Baleanu, X.-J. Yang, Local fractional variational iteration and decomposition methods for wave equation on Cantor sets within local fractional operators, Abstr. Appl. Anal., 2014 (2014), 6 pages.

[19] N. Baykus, M. Sezer, Solution of high-order linear Fredholm integro-differential equations with piecewise intervals, Numer. Methods Partial Differential Equations, 27 (2011), 1327-1339. 1

[20] W. S. Chung, Fractional Newton mechanics with conformable fractional derivative, J. Comput. Appl. Math., 290 (2015), 150-158. 1

[21] M. Ekici, M. Mirzazadeh, M. Eslami, Q. Zhou, S. P. Moshokoa, A. Biswas, M. Belic, Optical soliton perturbation with fractional-temporal evolution by first integral method with conformable fractional derivatives, Optik, 127 (2016), 10659-10669. 1

[22] A. M. A. El-Sayed, S. Z. Rida, A. A. M. Arafa, Exact solutions of fractional-order biological population model, Commun. Theor. Phys. (Beijing), 52 (2009), 992-996. 1

[23] A. Gökdoğan, E. Ünal, E. Çelik, Existence and uniqueness theorems for sequential linear conformable fractional differential equations, Miskolc Math. Notes, 17 (2016), 267-279. 1

[24] A. K. Golmankhaneh, X.-J. Yang, D. Baleanu, Einstein field equations within local fractional calculus, Rom. J. Phys., 60 (2015), 22-31. 1

[25] Z.-H. Guo, O. Acan, S. Kumar, Sumudu transform series expansion method for solving the local fractional Laplace equation in fractal thermal problems, Therm. Sci., 20 (2016), S739-S742. 1

[26] W. S. C. Gurney, R. M. Nisbet, The regulation of inhomogeneous populations, J. Theor. Biol., 52 (1975), 441-457. 1, 1

[27] M. E. Gurtin, R. C. MacCamy, On the diffusion of biological populations, Math. Biosci., 33 (1977), 35-49. 1, i, 1

[28] Z. Hacioglu, N. Baykus Savasaneril, H. Kose, Solution of Dirichlet problem for a square region in terms of elliptic functions, New Trends Math. Sci., 3 (2016), 98-103. 1

[29] H. Karayer, D. Demirhan, F. Büyükklç, Conformable fractional Nikiforov-Uvarov method, Commun. Theor. Phys. (Beijing), 66 (2016), 12-18. 1

[30] R. Khalil, M. Al Horani, A. Yousef, M. Sababheh, A new definition of fractional derivative, J. Comput. Appl. Math., 264 (2014), 65-70. 2.1, 2.2

[31] A. Kurt, Y. Çenesiz, O. Tasbozan, On the solution of Burgers equation with the new fractional derivative, Open Phys., 13 (2015), 355-360. 1

[32] Y.-Q. Liu, Z.-L. Li, Y.-Y. Zhang, Homotopy perturbation method to fractional biological population equation, Fract. Differ. Calc., 1 (2011), 117-124. 1

[33] Y.-G. Lu, Hölder estimates of solutions of biological population equations, Appl. Math. Lett., 13 (2000), 123-126. 1, 1, ii

[34] M. Ma, D. Baleanu, Y. S. Gasimov, X.-J. Yang, New results for multidimensional diffusion equations in fractal dimensional space, Rom. J. Phys., 61 (2016), 784-794. 1

[35] K. S. Miller, B. Ross, An introduction to the fractional calculus and fractional differential equations, A Wiley-Interscience Publication, John Wiley \& Sons, Inc., New York, (1993). 1

[36] A. Okubo, Diffusion and ecological problems: mathematical models, An extended version of the Japanese edition, Ecology and diffusion, Translated by G. N. Parker. Biomathematics, Springer-Verlag, Berlin-New York, (1980). iii, 1

[37] K. B. Oldham, J. Spanier, The fractional calculus, Theory and applications of differentiation and integration to arbitrary order, With an annotated chronological bibliography by Bertram Ross, Mathematics in Science and Engineering, Academic Press [A subsidiary of Harcourt Brace Jovanovich, Publishers], New York-London, (1974). 1

[38] I. Podlubny, Fractional differential equations, An introduction to fractional derivatives, fractional differential equations, to methods of their solution and some of their applications, Mathematics in Science and Engineering, Academic Press, Inc., San Diego, CA, (1999). 1

[39] P. Roul, Application of homotopy perturbation method to biological population model, Appl. Appl. Math., 5 (2010), 13691379. 1

[40] S. G. Samko, A. A. Kilbas, O. I. Marichev, Fractional integrals and derivatives, Theory and applications, Edited and with a foreword by S. M. Nikol'skil, Translated from the 1987 Russian original, Revised by the authors, Gordon and Breach Science Publishers, Yverdon, (1993). 1

[41] S. Sarwar, M. A. Zahid, S. Iqbal, Mathematical study of fractional-order biological population model using optimal homotopy asymptotic method, Int. J. Biomath., 9 (2016), 17 pages. 1

[42] N. B. Savasaneril, H. Delibas, Analytic solution for two-dimensional heat equation for an ellipse region, New Trends Math. Sci., 70 (2016), 65-70. 1

[43] F. Shakeri, M. Dehghan, Numerical solution of a biological population model using He's variational iteration method, Comput. Math. Appl., 54 (2007), 1197-1209. 1

[44] B. K. Singh, A novel approach for numeric study of 2D biological population model, Cogent Math., 3 (2016), 15 pages.

[45] J. Singh, D. Kumar, A. Kılıçman, Numerical solutions of nonlinear fractional partial differential equations arising in spatial diffusion of biological populations, Abstr. Appl. Anal., 2014 (2014), 12 pages.

[46] V. K. Srivastava, S. Kumar, M. K. Awasthi, B. K. Singh, Two-dimensional time fractional-order biological population model and its analytical solution, Egyptian J. Basic Appl. Sci., 1 (2014), 71-76. 1, 4.1, 4.1, 4.1, 1, 4.3, 4.3, 2, 4.3, 3, 4, $4.5,4.5,4.5,5,6,5$ 
[47] E. Ünal, A. Gökdoğan, Solution of conformable fractional ordinary differential equations via differential transform method, Optik, 128 (2017), 264-273. 1

[48] X.-J. Yang, Fractional derivatives of constant and variable orders applied to anomalous relaxation models in heat-transfer problems, Therm. Sci., 21 (2017), 1161-1171. 1

[49] X.-J. Yang, D. Baleanu, H. M. Srivastava, Local fractional similarity solution for the diffusion equation defined on Cantor sets, Appl. Math. Lett., 47 (2015), 54-60.

[50] X.-J. Yang, D. Baleanu, H. M. Srivastava, Local fractional integral transforms and their applications, Elsevier/Academic Press, Amsterdam, (2016).

[51] X.-J. Yang, F. Gao, H. M. Srivastava, New rheological models within local fractional derivative, Rom. Rep. Phys., 69 (2017), 12 pages.

[52] X.-J. Yang, H. M. Srivastava, C. Cattani, Local fractional homotopy perturbation method for solving fractal partial differential equations arising in mathematical physics, Rom. Rep. Phys., 67 (2015), 752-761.

[53] X.-J. Yang, J. A. Tenreiro Machado, A new fractional operator of variable order: application in the description of anomalous diffusion, Phys. A, 481 (2017), 276-283.

[54] X.-J. Yang, J. A. Tenreiro Machado, D. Baleanu, C. Cattani, On exact traveling-wave solutions for local fractional Korteweg-de Vries equation, Chaos, 26 (2016), 5 pages. 1 